\section{IgE-Tests von Alkoholkonsum beeinflusst}

\author{
Alkohol beeinflusst das Immunsystem in vielfältiger Weise. Welcher \\ Zusammenhang zwischen Alkoholkonsum und den Ergebnissen von \\ IgE-Tests besteht, untersuchte eine spanische Arbeitsgruppe.
}

A n der Studie nahmen 457 Erwachsene teil. 44 von ihnen konsumierten über $280 \mathrm{~g}$ Alkohol pro Woche (entspricht etwa drei Litern Rotwein). 195 der Teilnehmer tranken zwischen 10 und $280 \mathrm{~g}, 218$ konsumierten überhaupt keinen Alkohol. Zusätzlich untersuchten die Forscher noch 138 Alkoholiker, die stationär behandelt wurden. Sie führten einen Test auf spezifisches $\mathrm{IgE}$ gegen kreuzreaktive Kohlenhydratdeterminanten (,cross-reactive carbohydrate determinants" [CCD]), mehrere Pollen, Hymenopterengifte und Hausstaubmilbe durch. Kreuzreaktive CCD sind Oligosaccharide, die in pflanzlichen und tierischen Glykoproteinen vorkommen. Ihre klinische Bedeutung als Allergenepitope gilt als gering, problematisch ist jedoch die über sie vermittelte Kreuzreaktivität mit ande- ren Allergenen in IgE-Tests. In der Gesamtstudienpopulation wiesen 5,6 \% CCD-spezifisches IgE von mindestens $0,35 \mathrm{kU} / \mathrm{l}$ auf. Die Alkoholiker und die

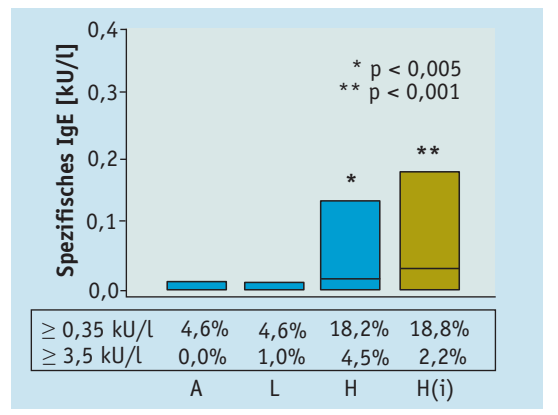

Das spezifische IgE gegen CCD ist bei Alkoholikern (Hi)) und höherem Alkoholkonsum $(H)$ signifikant erhöht im Vergleich zu abstinenten Personen (A) und solchen mit niedrigem bis moderatem Alkoholkonsum (L).
Studienteilnehmer mit hohem Alkoholkonsum zeigten signifikant höhere Antikörpertiter als die beiden anderen Gruppen. Auffällig war auch, dass spezifisches IgE gegen mehrere Pollen sowie Bienen- und Wespengift bei starkem Alkohoholkonsum oder Alkoholismus im Vergleich zu den beiden anderen Gruppen mit einer etwa doppelt so hohen Prävalenz auftrat. Alkoholiker zeigten zudem höhere Werte von spezifischem $\operatorname{IgE}$ gegen Hausstaubmilben.

Fazit: In der vorliegenden Studie zeigte sich, dass starker Alkoholkonsum mit gesteigerter Bildung zu höherem und häufigeren Nachweis von spezifischem IgE gegen CCD assoziiert war. Dies war verbunden mit vermehrtem Nachweis von IgE gegen Pollen oder Hymenopterengifte. Die Autoren raten, bei Patienten mit hohem Alkoholkonsum diese $\mathrm{Zu}$ sammenhänge bei der Bewertung von IgE-Tests zu berücksichtigen.

Gonzalez-Quintela A et al. Sensitization to cross-reactive carbohydrate determinants in relation to alcohol consumption. Clin Exp Allergy 2008; 38: 152-60

\title{
Weniger Kontaktallergien durch Alkohol?
}

\author{
Mehrere Studien zeigen ein erhöhtes Risiko für Allergien vom Sofort- \\ typ bei Menschen, die regelmäßig Alkohol trinken. Ob Alkohol auch \\ einen Effekt auf die Kontaktallergisierung hat, untersuchte eine Auto- \\ rengruppe im Rahmen der Glostrup Allergy Study.
}

nsgesamt 1.112 Jugendliche und Erwachsene im Alter zwischen 15 und 69 Jahren wurden 1990 zu ihrem Alkoholkonsum befragt. Gleichzeitig erfolgte bei den Studienteilnehmern ein standardisierter Atopiepatchtest mit 23 Allergenen. 1998 konnten 734 Studienteilnehmer nachuntersucht werden.

Allgemein wiesen signifikant mehr Frauen als Männer Kontaktallergien auf (1990: $23,2 \%$ vs. $10,4 \%$ ). Innerhalb von acht Jahren hatten sich bei $16 \%$ der Frauen neue Kontaktallergien entwickelt. Die entsprechende Inzidenz in diesem Zeitraum betrug bei Männern $8,2 \%$.
Zwischen Alkoholkonsum und der Sensibilisierungshäufigkeit auf Kontaktallergene bestand bei den männlichen Studienteilnehmern kein Zusammenhang. Dies galt für die Ersterhebung wie auch für die Nachfolgeuntersuchung. Die Tests bei den Frauen zeigten 1990 ebenfalls keine signifikante Assoziation von Alkoholkonsum und Prävalenz von Sensibilisierungen. Betrachtete man aber die bis zur zweiten Untersuchung neu aufgetretenen Kontaktallergien, so entwickelten Frauen, die überhaupt keinen Alkohol tranken, häufiger Sensibilisierungen als solche, die Alkohol konsumierten. Die Odds Ratio zeigte dabei ein um den Faktor 2,12 erhöhtes Risiko. Die Autoren werteten dies jedoch nur als statistischen Trend. Einen $\mathrm{Zu}$ sammenhang zwischen der Menge des konsumierten Alkohols und der geringeren Inzidenz von Sensibilisierungen gab es in dieser Untersuchung nicht.

Fazit: Die Studie aus Dänemark ergab einen deutlichen Trend für ein vermehrtes Auftreten von Kontaktallergien bei Frauen, die keinen Alkohol trinken. Dieses Ergebnis galt aber nur für die Inzidenz von Neusensibilisierungen innerhalb eines Zeitraums von acht Jahren. Bei Männern zeigte sich weder für die Prävalenz, noch für die Inzidenz von Kontaktallergien ein Zusammenhang mit dem Alkoholkonsum.

Thyssen JP et al. The association between alcohol consumption and contact sensitization in Danish adults: the Glostrup Allergy Study. Br J Dermatol 2008; 158 : 306-12 